\title{
étude expérimentale du comportement d'une vase sous chargement cyclique
}

\author{
par \\ A. Pecker \\ Ingénieur, à la Société Mécasol, Paris - \\ Maître de Conférences à l'École Nationale des Ponts et Chaussées. \\ J.-M. Dupas \\ Directeur général de la Société Mécasol, Paris - \\ Maître de Conférences à l'École Nationale des Ponts et Chaussées.
}

La nécessité de dimensionner des ouvrages importants, comme les centrales nucléaires vis-à-vis des risques sismiques ou les plates-formes en mer vis-àvis des sollicitations dues à la houle, requiert la connaissance du comportement des sols de fondation sous l'effet de chargements cycliques. Dans la nature, ces chargements sont multidirectionnels et d'amplitude variable dans le temps. Même si un matériel de laboratoire sophistiqué permet théoriquement la reproduction de ces sollicitations, il est d'usage d'étudier le comportement du matériau sous chargement unidirectionnel d'amplitude constante (essai à déformation contrôlée) et de transposer les résultats obtenus aux sollicitations réelles. Une telle approche limite les données de l'étude, et permet de mieux en apprécier les paramètres fondamentaux.

La recherche faite porte sur le comportement d'une vase soumise au laboratoire à des sollicitations cycliques unidirectionnelles imposant des chemins de contrainte variables suivant le type d'essai réalisé. On s'est attaché plus particulièrement à la vérification de la validité des lois de comportement habituellement utilisées et aux modifications à apporter à ce type de loi pour tenir compte du phénomène de dégradation des caractéristiques sous sollicitation dynamique comportant de nombreux cycles de charge.

\section{Rappel sur les lois de comportement des sols sous chargement cyclique}

Un échantillon de sol soumis au laboratoire à des cycles de chargement cyclique présente une courbe effort déformation analogue à celle de la figure 1. On distingue une courbe de premier chargement (trait tireté) et l'apparition, lors de décharges et recharges successives, d'une boucle d'hystérésis. Plus la déformation maximale atteinte au cours du cycle augmente. plus le module de cisaillement sécant (pente de la droite joignant l'origine à l'extrémité de la boucle) diminue. Cela signifie que sous chargement cyclique, tout comme sous chargement statique unidirectionnel, le sol a un comportement non linéaire. L'aire de la boucle d'hystérésis, qui caractérise l'énergie dissipée par le matériau au cours du chargement, augmente lorsque la déformation maximale atteinte au cours du cycle augmente. Enfin, on constate expérimentalement que la forme de la boucle d'hystérésis ne dépend pas de la fréquence de la sollicitation appliquée, ce qui signifie que l'amortissement du sol est de nature purement hystérétique et non d'origine visqueuse.

Pour décrire ce type de comportement, les modèles les plus couramment utilisés sont brièvement décrits ci-dessous (Pecker 1979).

\section{1 Élasticité linéaire}

Le sol est représenté par un seul ressort défini par sa constante $\mathrm{G}$ représentant le module de cisaillement. La loi de comportement s'écrit :

$$
\tau=\mathrm{G} \gamma
$$

avec $\tau=$ contrainte de cisaillement cyclique

$$
\gamma=\text { déformation de cisaillement cyclique. }
$$

D'après les constatations expérimentales, ce type de loi ne peut représenter qu'imparfaitement le comportement du sol sous chargement cyclique. Cependant pour tous les problèmes de vibration de machine où les déformations induites dans le sol sont de très faible amplitude, la loi de l'élasticité linéaire constitue une approximation tout à fait satisfaisante.

\subsection{Modèle visco-élastique linéaire}

Le sol est représenté par un ressort de caractéristique de raideur $\mathrm{G}$ et par un amortissement de coefficient $\eta$ placé en parallèle (modèle de Kelvin-Voigt). La loi de comportement s'écrit :

$$
\tau=\mathrm{G} \gamma+\eta \dot{\gamma} .
$$

oủ $\gamma$ et $\dot{\gamma}$ représentent la déformation et la vitesse de déformation. 
Dans ce type de modélisation, le coefficient $\eta$ est en général pris constant, ce qui correspond à un amortissement visqueux. Pour tenir compte du comportement hystérétique du sol, il est possible d'introduire, selon une procédure décrite par Jacobsen (1930), un amortissement visqueux dit "équivalent " permettant de ramener l'étude d'un matériau hystérétique à celui d'un matériau visco-élastique. Dans cette procédure, l'équivalence se fait sur l'égalité des amplitudes à résonance du modèle visco-élastique et du modèle hystérétique. On définit ainsi un pourcentage d'amortissement critique équivalent $\beta$ :

$$
\beta=\frac{1}{4 \pi} \frac{\Delta w}{w}=\frac{1}{2 \pi} \frac{\Delta w}{G_{s} \gamma_{\max }^{2}}
$$

où $\Delta w$ (aire de la boucle) = énergie totale dissipée pendant le cycle.

$w=$ énergie élastique emmagasinée par le système au cours du cycle.

L'introduction d'un module de cisaillement complexe $\mathrm{G}^{*}$ permet d'écrire la loi de comportement sous la forme :

$$
\tau=G^{*} \gamma .
$$

Le module $G^{*}$ est fonction des caractéristiques $G$ et $\beta$ du matériau. Le modèle de ce type le plus couramment utilisé est celui développé par Lysmer (1975) :

$$
G^{*}=G_{s}\left[1-2 \beta^{2}+2 i \beta \sqrt{1-\beta^{2}}\right]
$$

Mis en œuvre avec un processus itératif permettant de prendre en compte de façon approchèe la non linéarité du sol, ce type de modèle est d'utilisation simple. Il permet de calculer de façon satisfaisante les accélérations et contraintes maximales induites. II surestime l'amortissement dû aux hautes fréquences (Martin 1975) et ne permet pas de calculer les déformations permanentes résultant d'une sollicitation cyclique. Celles-ci ne peuvent être appréciées qu'à l'aide de modèles purement non linéaires.

\subsection{Modèles non linéaires}

Il en existe un grand nombre développés au cours de ces dernières années. Leur description n'entre pas dans le cadre de cette étude. On pourra se reporter à la revue générale faite par Hardin (1978). La plupart des modèles non linéaires découlent des deux lois de Masing (1926), qui postulent que :

- les déformations irréversibles du sol sont d'origine purement plastique. A chaque inversion du signe du chargement, le matériau retrouve ses propriétés ini- tiales. En d'autres termes, le module tangent à la boucle d'hystérésis, juste après le changement de sens du chargement, est égal au module tangent initial à la courbe de premier chargement (fig. 1);

- les courbes de recharge et décharge successives se déduisent de la courbe de premier chargement par une dilatation d'un facteur 2 des échelles horizontale et verticale.

D'un point de vue rhéologique, Iwan (1967) a montré qu'un matériau satisfaisant aux lois de Masing peut être représenté par un ensemble de ressorts et de frotteurs (fig. 2).

Les lois de comportement traduisant de façon mathématique ces deux lois, peuvent prendre des formes diverses (Martin 1975) suivant que l'on exprime la contrainte en fonction de la déformation (modèle de Davidenkoff) oú la déformation en fonction de la contrainte (modèle de Ramberg-Osgood). Dans la présente étude, la vérification de la validité des lois de Masing a été faite à partir de la formulation de Ramberg-Osgood, qui prend la forme :

$$
\begin{gathered}
y=f(\tau)=\gamma_{c}+\frac{1}{G_{\max }}\left(\tau-\tau_{c}\right)\left[1+H\left(\frac{\left|\tau-\tau_{c}\right|}{n}\right)\right] \\
H(x)=\alpha\left[\frac{x}{\tau_{y}}\right]^{R-1}
\end{gathered}
$$

avec $\tau_{c}$ et $\gamma_{c}=$ valeurs de la contrainte et de la déformation lors de la précédente inversion du cycle du chargement.

$n$ is paramètre de valeur 1 sur la courbe de premier chargement et 2 sur les courbes de décharge et recharge successives.

$\mathrm{G}_{\max }=$ module de cisaillement maximal du sol (pente de la tangente à la courbe de premier chargement au voisinage de l'origine).

$\approx$ et $\mathrm{R}=$ constantes caractéristiques du matériau. $\tau_{y}=$ contrainte de cisaillement de référence.

La loi de comportement implique la connaissance de 4 paramètres : $G_{\max }, \tau_{y}$, $x$ et $R$. Seul le paramètre $G_{\max }$ peut être déterminé à partir d'essai in-situ. La connaissance des autres paramètres nécessitent la réalisation d'essais de laboratoire sous des conditions de contraintes ou déformations contrôlées et pour des sollicitation d'amplitudes très faibles à grandes.

\section{Caractéristiques du sol utilisé}

Les échantillons testés pour les besoins de la présente étude proviennent du site du Blayais. II s'agit de vases prélevées à une profondeur de $7 \mathrm{~m}$ à $10 \mathrm{~m}$. Ses caractéristiques d'identification, mécaniques et de compressibilité sont rassemblées sur le tableau I. II

Tableau I Caractéristiques mécaniques de la vase du Blayais

Profondeur de prélèvement

Teneur en eau naturelle

Poids volumique apparent sec

Poids volumique des particules solides

Limites d'Atterberg (étuvage à $105^{\circ} \mathrm{C}$ )

Caractéristiques mécaniques apparentes

Caractèristiques intergranulaires

Pression de consolidation

Indice de compression

Indice de gonflement

Coefficient de consolidation charge

Coefficient de consolidation décharge

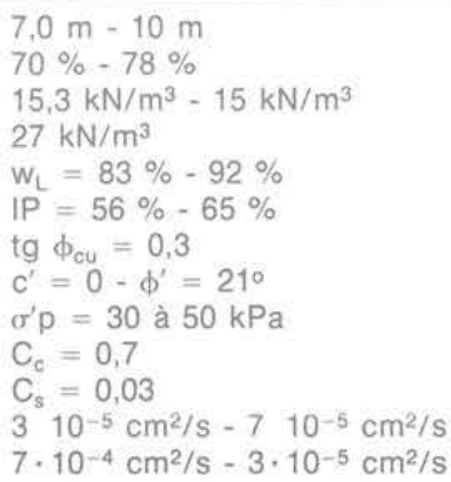




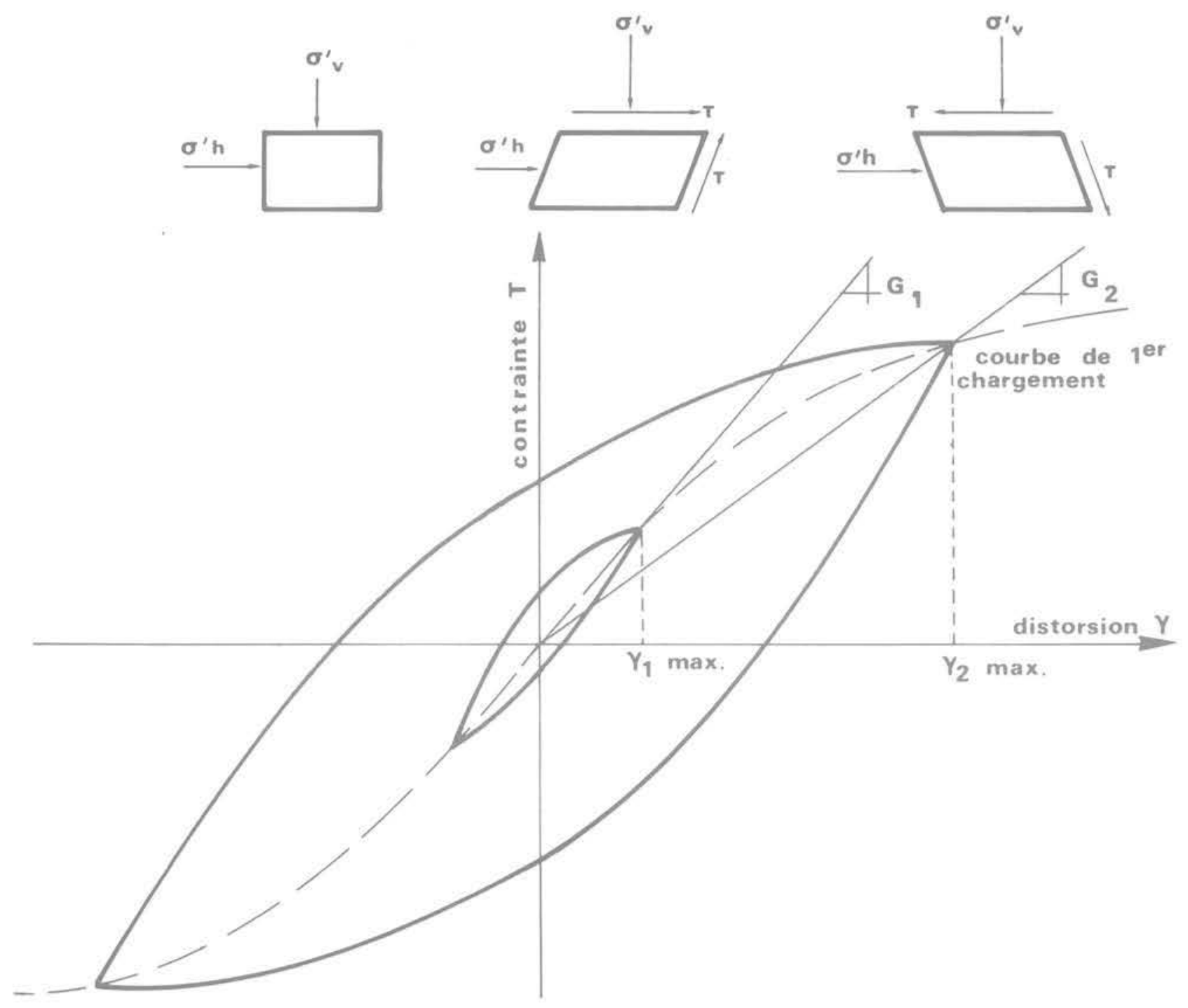

Fig. 2 Modèle plastique
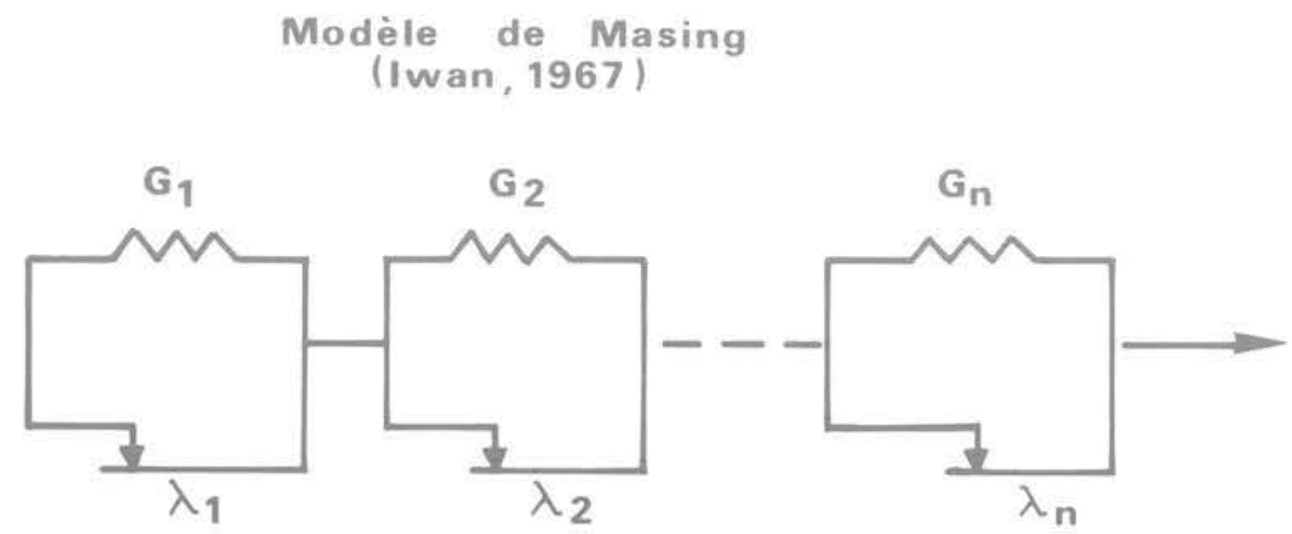

Fig. 1 Courbe effort - Déformation cyclique 
s'agit d'une argile de très forte plasticité dans la classification de Casagrande. Sa teneur en eau naturelle est élevée et son poids volumique apparent sec faible. On peut retenir comme loi d'augmentation de la cohésion apparente $c_{u}$ avec l'étreinte de consolidation $\sigma_{m}^{\prime}$ :

$$
\Delta \mathrm{c}_{\mathrm{u}}=0,3 \Delta \sigma_{\mathrm{m}}^{\prime}
$$

Les caractéristiques intergranulaires sont : $c^{\prime}=0$, $\Phi^{\prime}=21^{\circ}$ (valeurs en bon accord avec l'indice de plasticité).

Les essais œdométriques traduisent un sol normalement consolidé, fortement compressible au-delà de sa pression de consolidation $(\mathrm{Cc}=0,7)$ et peu gonflant en décharge. Les coefficients de consolidation varient avec la contrainte appliquée de $310^{-5} \mathrm{~cm}^{2} / \mathrm{s}$ à $7 \cdot 10^{-5} \mathrm{~cm}^{2} / \mathrm{s}$ au chargement et de $7 \cdot 10^{-4} \mathrm{~cm}^{2} / \mathrm{s}$ à $310^{-5} \mathrm{~cm}^{2} / \mathrm{s}$ au déchargement. Compte tenu des contraintes appliquées aux éprouvettes, on a retenu pour la présente étude un coefficient de consolidation égal à $510^{-5} \mathrm{~cm}^{2} / \mathrm{s}$ au chargement et à $10^{-4} \mathrm{~cm}^{2} / \mathrm{s}$ au déchargement.

Les limites d'Atterberg sur échantillons étuvés à $60^{\circ}$ sont notablement différentes de celles mesurées sur échantillons étuvés à $105^{\circ}$, ce qui traduit une teneur élevée en matière organique. En toute rigueur, les teneurs en eau mesurées dans ces conditions ne sont pas représentatives de la quantité d'eau libre présente dans l'échantillon. II n'en a cependant pas été tenu compte dans cette étude.

\section{Les essais de laboratoire}

La détermination des paramètres intervenant dans la loi de comportement nécessite la réalisation d'essais de laboratoire couvrant la plus grande plage possible de déformations. Le paramètre $G_{\max }$ nécessite des mesures à très faible déformation, et les paramètres $\alpha$ et $R$ des mesures à forte déformation. En effet, d'après les équations (6) et (7) l'expression asymptotique de la courbe de premier chargement s'écrit (pour $R=1$ ):

$$
\gamma \sim \frac{\alpha}{G_{\max } \tau_{y}^{R-1}} \tau^{R}
$$

Sur un graphique logarithmique, la relation entre $y$ et $\tau$ est une droite de pente R. Pour $T=\tau_{y}$. on a $\gamma=\left(x \tau_{y} / G_{\max }\right.$

\subsection{Essai de colonne résonnante}

Ce type d'essai permet la mesure du module de cisaillement à très petite déformation. Après consolidation isotrope sous une étreinte donnée et application de la contre-pression nécessaire à sa saturation. l'éprouvette est mise en vibration forcée, La vibration peut être longitudinale ou transversale.

La fréquence est modifiée jusqu'à l'obtention de la résonance. La fréquence de résonance est reliée au module $\mathrm{G}_{\mathrm{s}}$ et aux caractéristiques géométriques de l'éprouvette (Richart et al 1970). Il est également possible d'obtenir avec l'appareillage disponible à Mécasol, les boucles d'hystérésis du sol pour des déformations de $10^{-6}$ à $10^{-3}$ environ.

Les études de Hardin et Black (1968) ont montré que le module de cisaillement maximal $\mathrm{G}_{\max }$ ne dépend pratiquement que de la contrainte moyenne de consolidation $\sigma_{m}^{\prime}$ et de l'indice des vides e. L'influence de ce dernier paramètre, qui est par ailleurs moindre, n'a pas été mise en évidence car, du fait de la procédure d'essais choisie, il existe une relation bi-univoque entre l'indice des vides (ou la teneur en eau, les échantillons étant saturés) et la contrainte moyenne de consolidation. Pour déterminer la loi de variation de $\mathrm{G}_{\max }$ d'un sol normalement consolidé avec l'étreinte de consolidation, les essais ont été réalisés sous 5 étreintes de consolidation $(0,1$ à $0,5 \mathrm{MPa})$ nettement supérieures au poids des terres.

\subsection{Essai triaxial dynamique}

II permet avec l'appareillage disponible, la dètermination des caractéristiques de déformation dynamique (module et amortissement) pour des déformations de $10^{-4}$ à $10^{-2}$ environ. Après consolidation isotrope sous une étreinte donnée et application de la contrepression nécessaire à sa saturation, l'échantillon est soumis à une déformation axiale d'amplitude : $+\varepsilon$, $-\varepsilon$. On enregistre directement les boucles d'hystérésis dans le plan $(E, \varepsilon)$. La connaissance du coefficient de Poisson (égal à 0,5 pour un sol parfaitement saturé et non drainé) permet de convertir ces résultats dans le plan $(\tau, \gamma)$.

Les essais triaxiaux dynamiques ont été réalisés aux mêmes étreintes de consolidation que les essais de colonne résonnante. A chaque niveau de la déformation. l'échantillon est soumis au plus à 3 cycles pour éviter l'apparition du phénomène de fatigue.

\section{Résultats des essais}

\subsection{Module de cisaillement maximal}

Le résultat d'un des essais de colonne résonnante, effectué sous une étreinte de consolidation de $0.5 \mathrm{MPa}$, est représenté (fig. 3 ) sous forme de la variation du module de cisaillement sécant $\mathrm{G}_{\mathrm{s}}$ (pente de la droite joignant l'origine aux extrémités de la boucle d'hystérésis) en fonction de la déformation maximale atteinte au cours du cycle. Pour des déformations inférieures à $10^{-4}$ environ, le module ne varie pratiquement pas avec la déformation, et le comportement du sol est linéaire. Pour des déformations supérieures à $10^{-4}$, le module diminue fortement quand la déformation augmente. Pour une déformation de $10^{-3}$, sa diminution est de $30 \%$. Rappelons que les déformations induites par des séismes importants sont supérieures à $10^{-3}$.

La figure 4 regroupe les valeurs des modules de cisaillement maximaux en fonction des étreintes de consolidation. II existe une bonne corrélation entre module et étreinte de consolidation; on peut valablement retenir la loi :

$$
\mathrm{G}_{\max }=210 \mathrm{p}_{\mathrm{a}}\left[\frac{\sigma_{\mathrm{m}}^{\prime}}{\mathrm{p}_{\mathrm{a}}}\right]^{0,9}
$$

avec $p_{a}=$ pression atmosphérique.

D'après l'équation (10) le module de cisaillement maximal est pratiquement proportionnel à la contrainte moyenne de consolidation $\sigma_{m}$ (exposant 0.9 ). Le sol étant normalement consolidé, il y a proportionnalité entre sa cohésion non drainée et sa contrainte de consolidation. Le module de cisaillement maximal $G_{\max }$ est donc pratiquement proportionnel à la cohésion non drainée. 


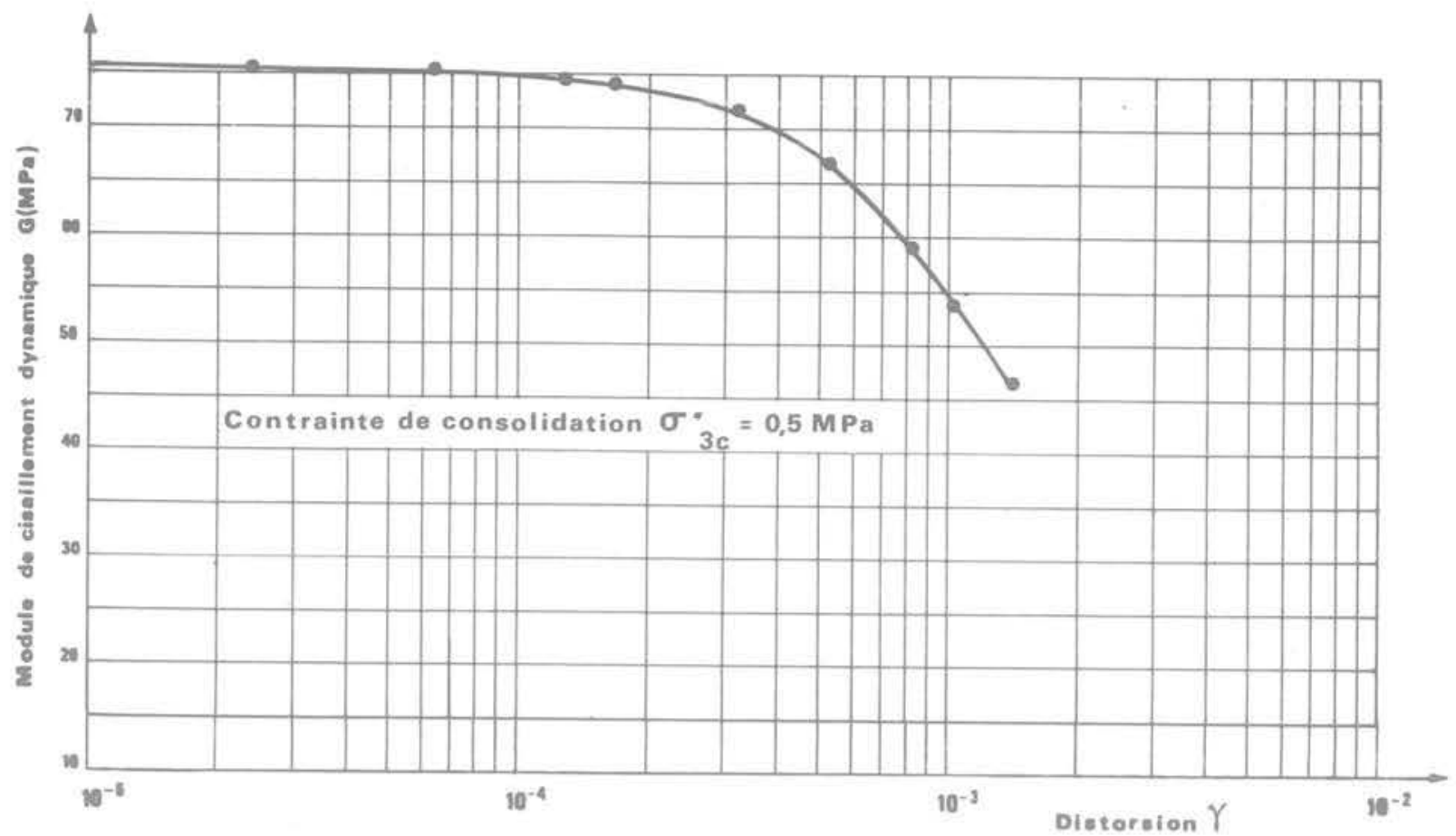

Fig. 3 Essai à la colonne résonnante

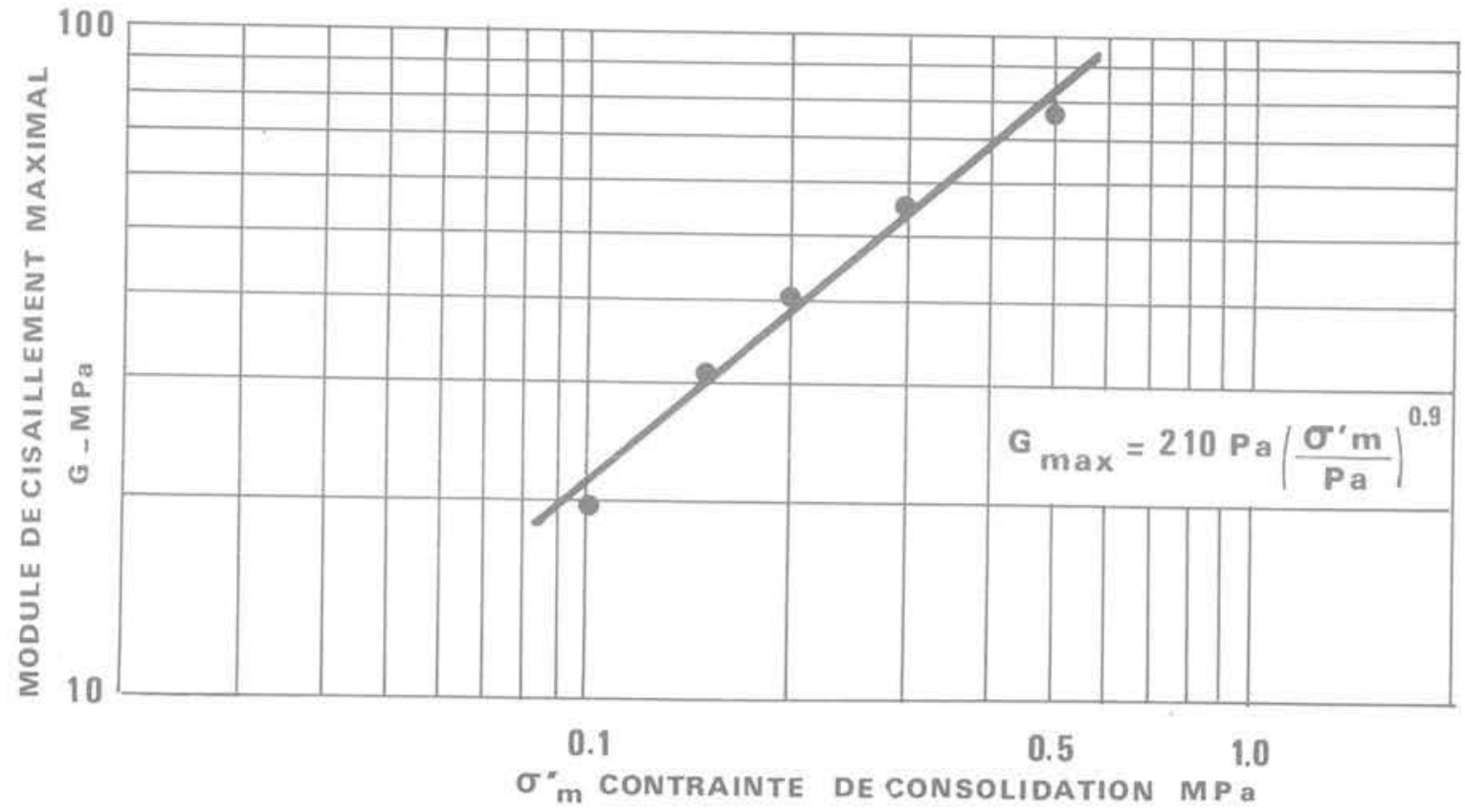

Fig. 4 Essai à la colonne résonnante - Variation du module maximal avec l'étreinte de consolidation 


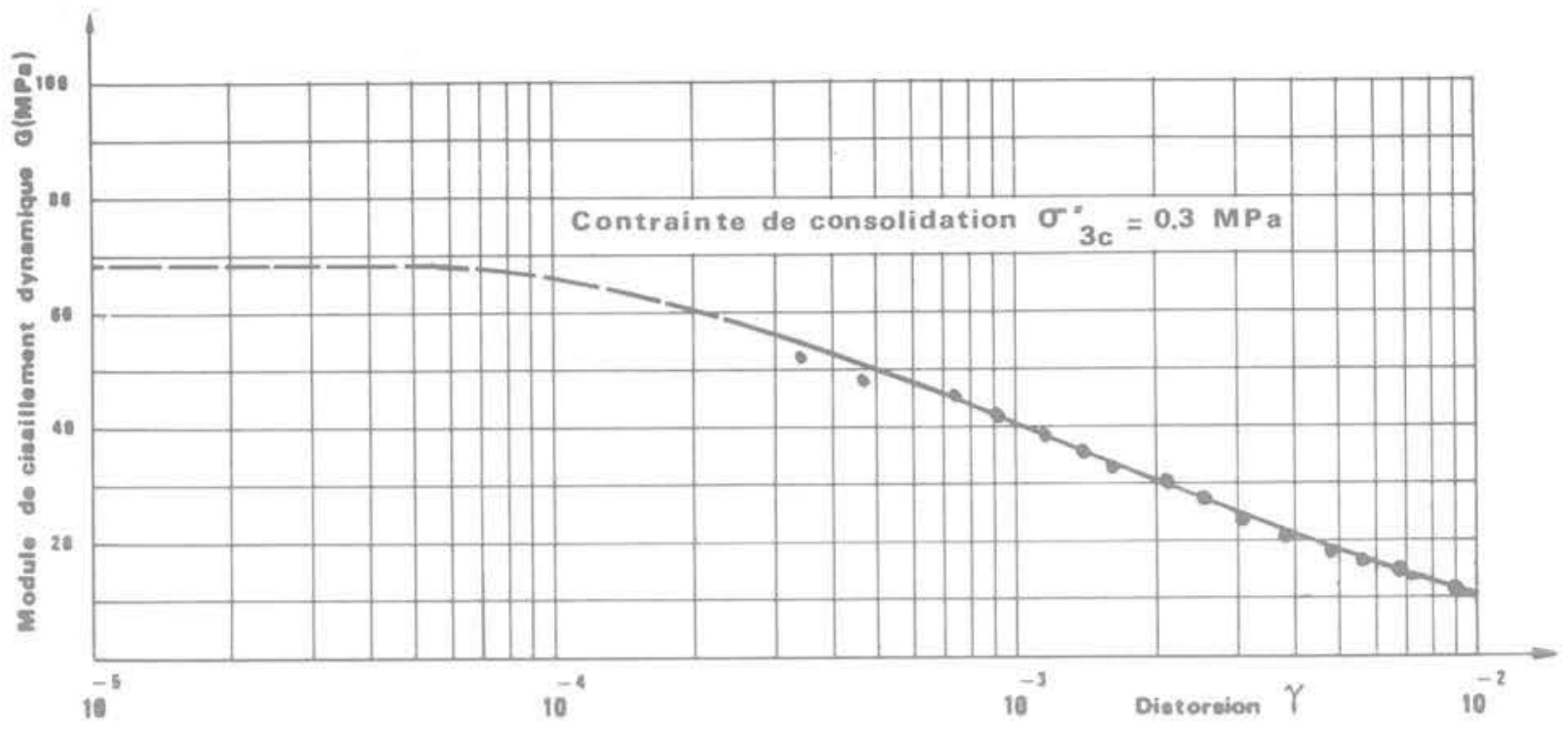

Fig. 5 Essai de cisaillement dynamique à I'appareil triaxial

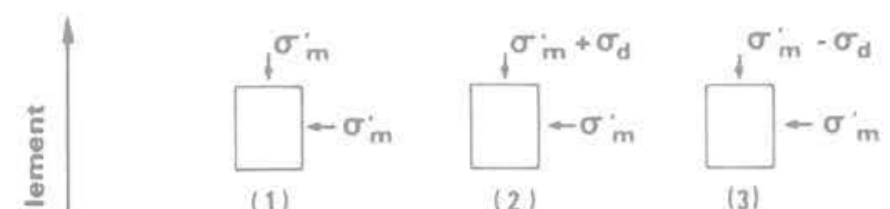

(1) (2) (3)

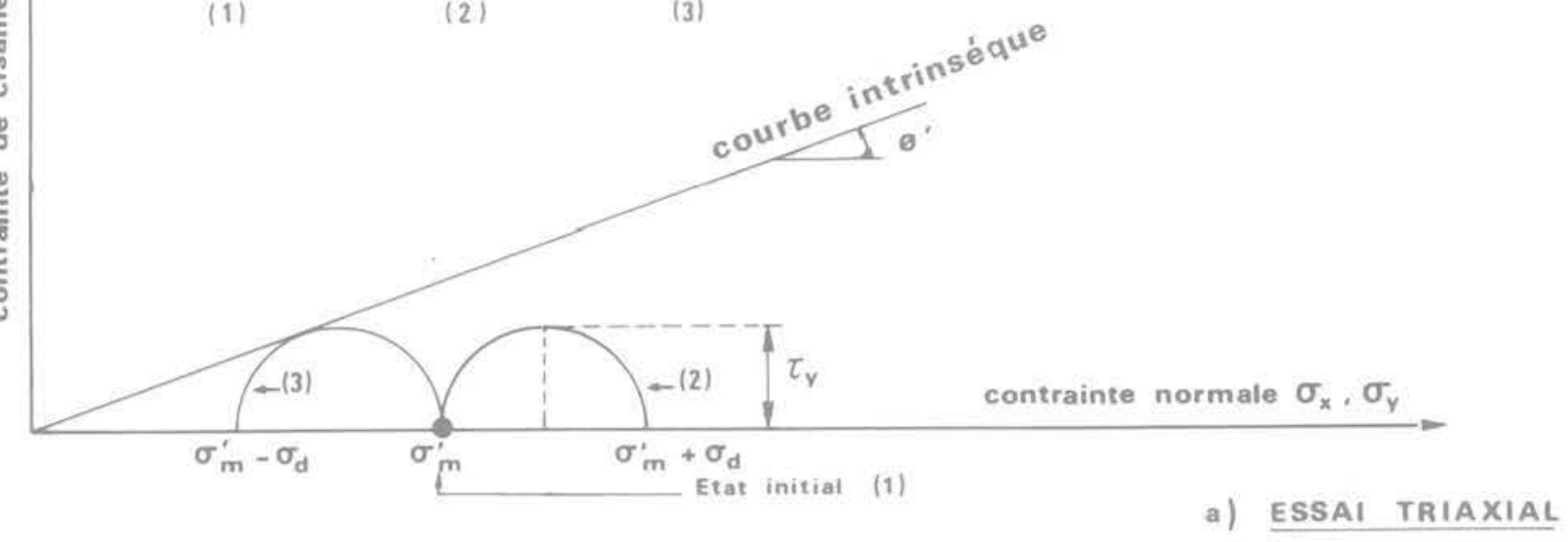

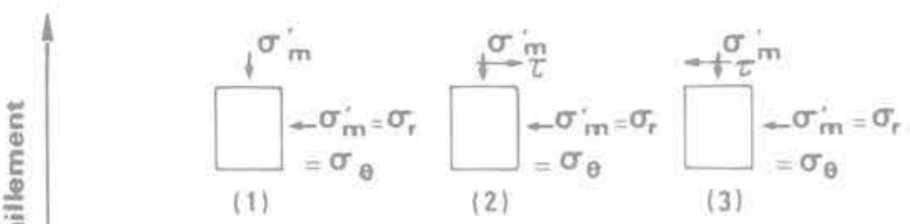

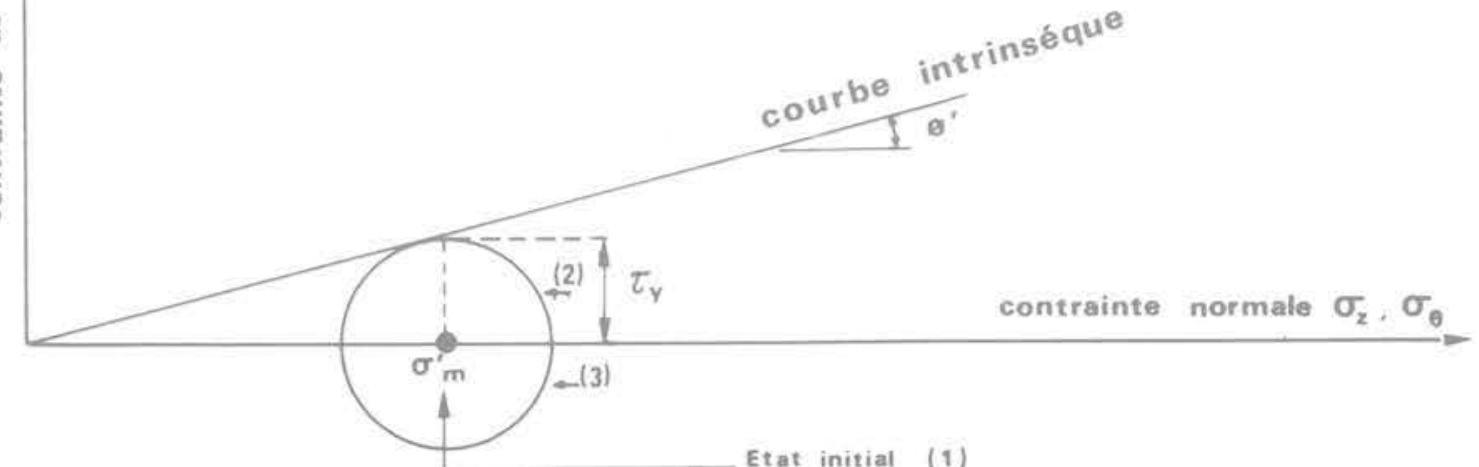

b) COLONNE RESONNANTE

Fig. 6 Chemin de contrainte suivi dans les essais dynamiques 


\subsection{Paramètres $\alpha$ et $R$}

Le résultat d'un essai triaxial dynamique réalisé sous une étreinte de consolidation de $0,3 \mathrm{MPa}$ et ayant servi à la détermination des paramétres $\alpha$ et $\mathrm{R}$ est donné sur la figure 5 . La décroissance du module $G_{s}$ est importante pour les déformations supérieures à $10^{-3}$. Pour une déformation de $10^{-2}$, le module est égal à $0,15 G_{\max }\left(G_{\max }\right.$ étant obtenu par extrapolation). A partir d'une série d'essais réalisés à des étreintes variant de 0,1 à $0.5 \mathrm{MPa}$, on a obtenu $\alpha=3$. $\mathrm{R}=2,8$.

\subsection{Contrainte de référence $\tau_{y}$}

Le module de cisaillement $\mathrm{G}_{\mathrm{s}}$ étant a priori fonction du chemin de contrainte, il a été admis que ce paramètre n'influait que sur $\tau_{y}$. Les chemins de contrainte a l'appareil triaxial dynamique et à l'appareil de colonne résonnante sont représentés sur la figure 6 . On a choisi de prendre comme valeur de $\tau_{y}$ la valeur de la contrainte de cisaillement maximale susceptible d'être appliquée à l'échantillon, indépendamment du plan sur lequel elle s'exerce. Son expression s'obtient aisément à partir des caractéristiques intergranulaires :

à l'appareil triaxial :

$$
\tau_{y}=\frac{\sigma_{m}^{\prime} \sin \phi^{\prime}}{1+\sin \phi^{\prime}}
$$

à la colonne résonnante :

$$
\tau_{y}=\sigma_{m}^{\prime} \sin \phi^{\prime} .
$$

\section{Validité du modèle de Ramberg-Osgood}

Pour tous les essais réalisés, on a rassemblé sur la figure 7. la variation, déduite de l'esssai triaxial dynamique, du module de cisaillement secant avec la déformation et, sur la figure 8 , les mêmes éléments déduits de l'essai de colonne résonnante. Les courbes expérimentales sont représentées en traits pleins. Les courbes théoriques calculées à partir du modèle et des valeurs des paramètres déterminées expérimentalement sont représentées en tiretés. II y a bon accord entre les mesures et le modèle théorique. L'écart maximal correspond à l'étreinte de consolidation de $0.1 \mathrm{MPa}$; il ne dépasse pas $10 \%$ à $15 \%$. Cet accord montre également que l'influence du chemin de contrainte sur la valeur du module a été correctement prise en compte avec le seul paramètre $\tau_{y}$. On en déduit que le modèle de Ramberg-Osgood permet de définir correctement la courbe de premier chargement.

Pour s'assurer de la complète validité du modèle, il faut également s'assurer qu'il permet de définir correctement I'hystérésis. On a représenté (fig. 9 et 10) les boucles d'hystérésis mesurées et calculées pour deux niveaux de déformation $\left(810^{-4}\right.$ et $\left.7 \cdot 10^{-3}\right)$. Ces résultats, caractéristiques de l'ensemble des essais, montrent que :

- les extrémités des boucles d'hystérésis mesurées et calculées coinncident correctement quelle que soit la déformation maximale. Ceci confirme que le modèle permet de définir correctement la courbe de premier chargement.

- à faible déformation, il y a bonne concordance sur presque toute la boucle entre les valeurs mesurées et calculées.

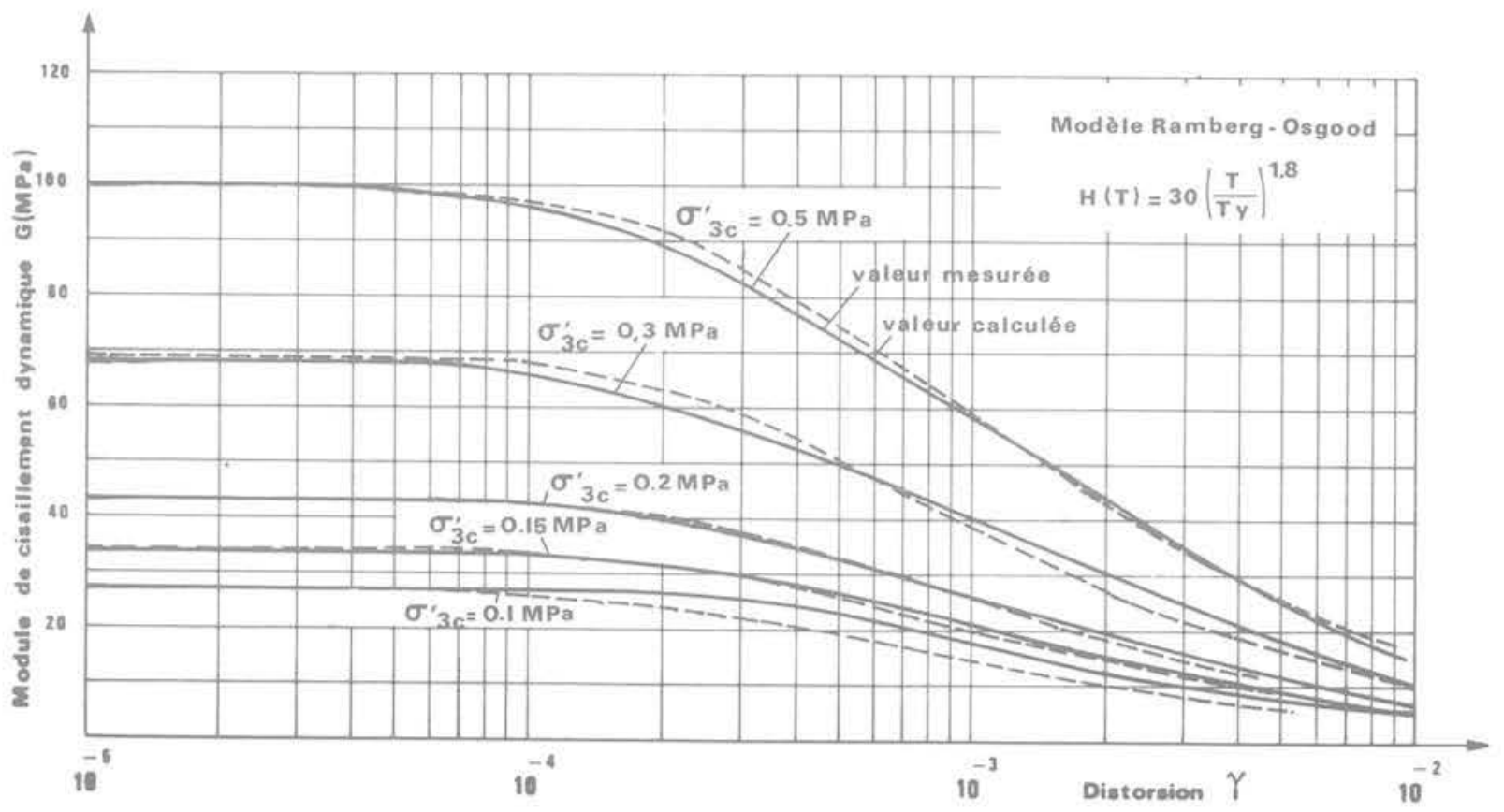

Fig. 7 Essais de cisaillement dynamique à l'appareil triaxial 


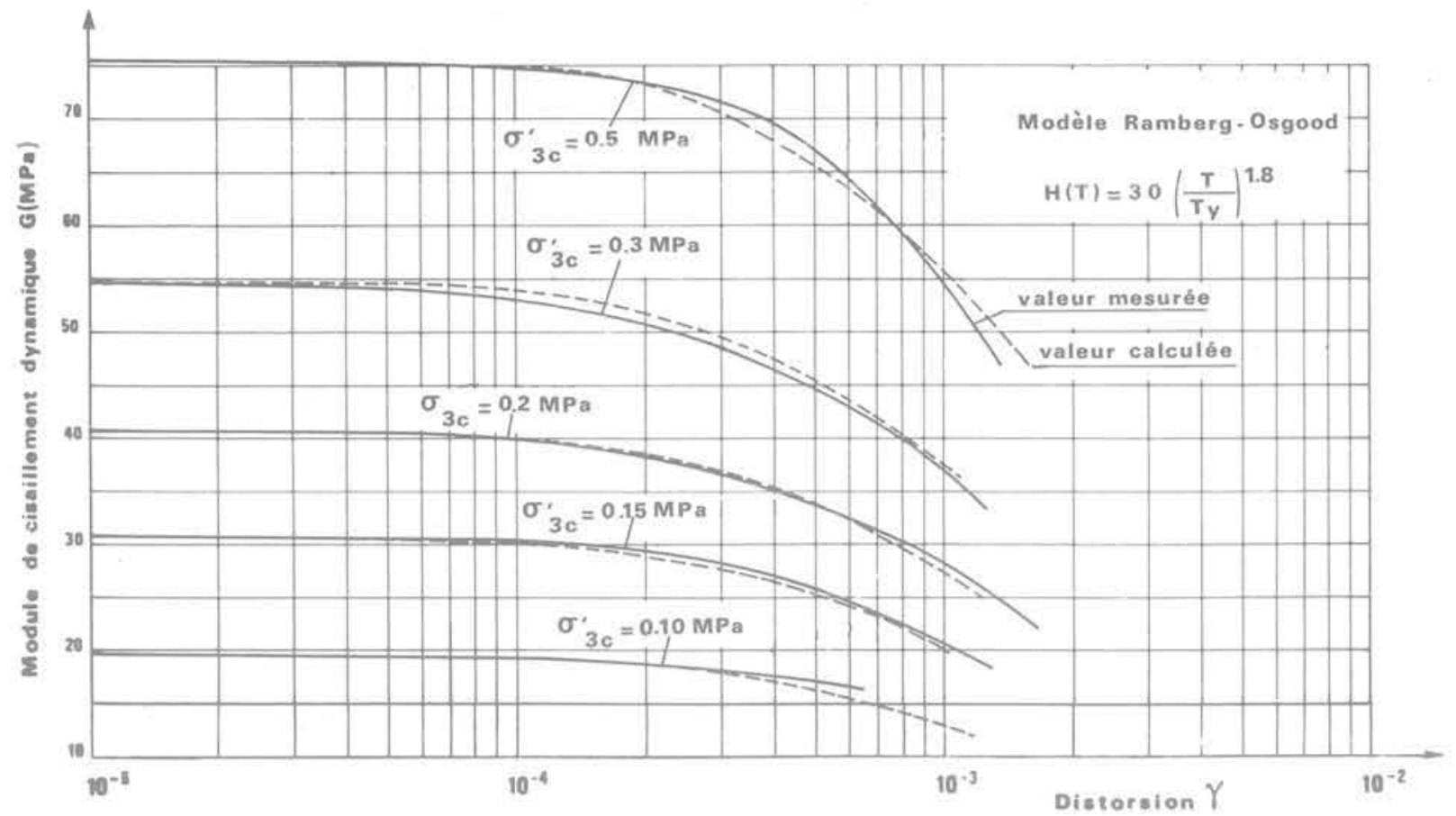

Fig. 8 Essais à la colonne résonnante

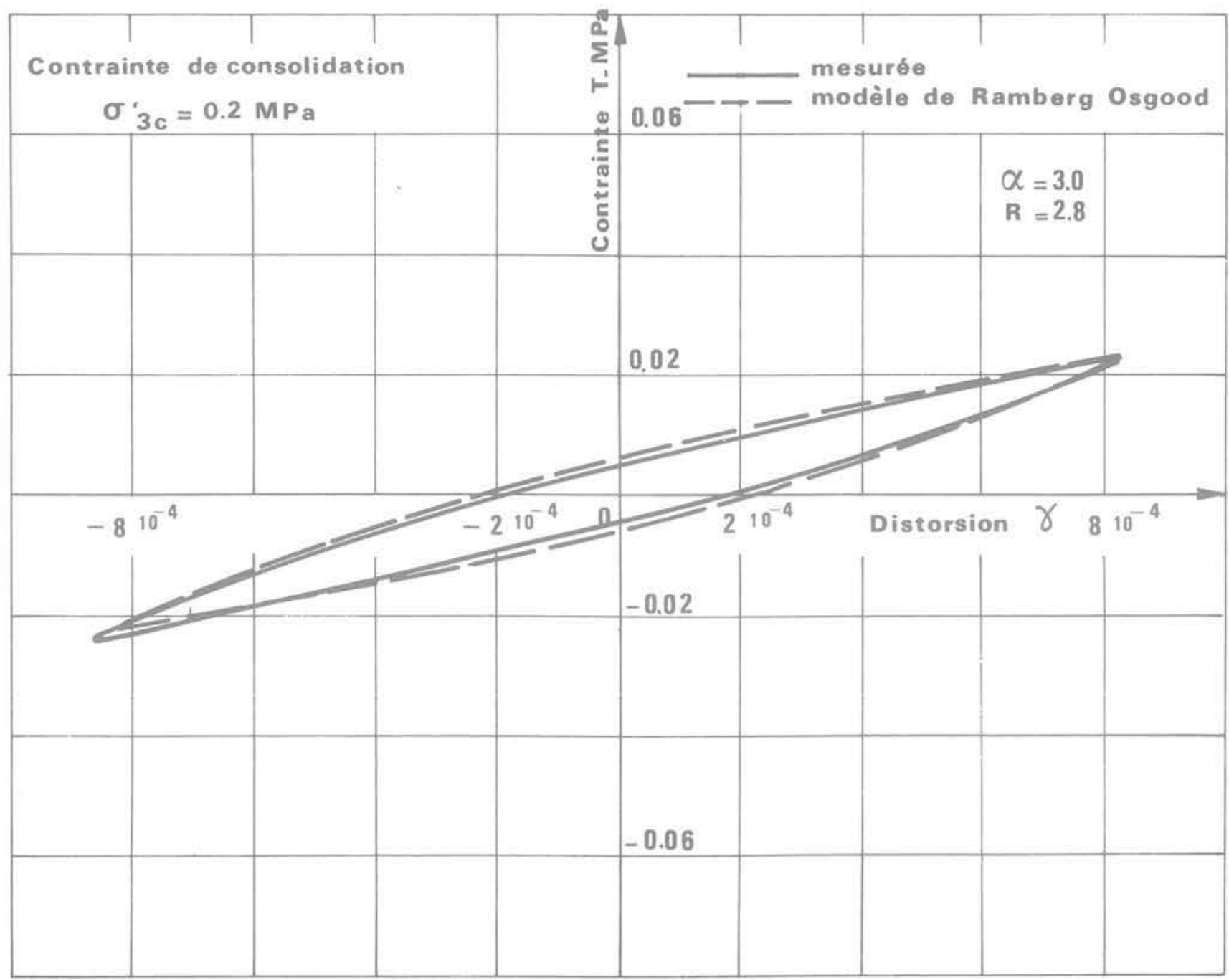

Fig. 9 Courbe d'hystérésis mesurée à l'appareil triaxial 
- à forte déformation, la boucle d'hystérésis calculée est plus importante que celle mesurée. Le modèle de Ramberg-Osgood, et par conséquent les lois de Masing. surestime l'énergie interne dissipée. D'un point de vue pratique, ceci a vraisemblablement peu de conséquences car, dans les problèmes d'interaction sol structure, l'énergie dissipée par radiation (amortissement géométrique) est beaucoup plus élevée que celle dissipée par hystérésis. D'un point de vue théorique, on pourrait cependant envisager de modifier la seconde loi de Masing en dilatant les échelles des contraintes et déformations des courbes de déchargement-rechargement par un facteur $\mathrm{x}$ variable avec le niveau de déformation maximale atteint. Ce facteur serait égal à 2 à faible déformation (Loi de Masing) et diminuerait à forte déformation. Le programme d'essais réalisés ne permet pas de préciser les valeurs à donner à $\mathrm{x}$.

\section{Comportement sous chargement cyclique de longue durée}

Des études antérieures pour les fondations de platesformes en mer du Nord ont montré que le module de cisaillement sécant du sol diminue lorsque le nombre de cycles de sollicitation augmente. Pour permettre une meilleure compréhension de ce phénomène, des essais dits "de fatigue " ont été réalisés à l'appareil triaxial dynamique, suivant le processus opératoire décrit ci-dessus, mais avec un nombre de cycles de sollicitation élevé. Les essais ont été réalisés sous 4 étreintes de consolidation $(0,1$ à $0,5 \mathrm{MPa})$. Pour chaque étreinte, un effort constant cyclique est appliqué pendant 1000 cycles, et la variation de $G_{s}$ en fonction du nombre de cyles est calculée. Le drainage restant fermé, l'effort appliqué est augmenté puis une nouvelle série de 1000 cycles est appliquée, etc. Pour chaque éprouvette, il a été réalisé 3 à 5 séries de 1000 cycles. Les efforts sont choisis de façon à représenter une fraction constante $(10 \%$ à $50 \%)$ de la cohésion non drainée. Après la dernière série de 1000 cycles, l'échantillon est cisaillé à drainage fermé pour mesurer sa cohésion apparente. Après la fin de la consolidation, le drainage n'est jamais ouvert.

Les résultats d'un de ces essais, correspondant à une étreinte de consolidation de $0,5 \mathrm{MPa}$, sont représentés sur la figure 11. Pour les faibles efforts cycliques, la dispersion des mesures est parfois importante; elle peut être attribuée à l'appareillage, l'asservissement de la contrainte à de très faibles valeurs étant difficile à réaliser. Le module diminue lorsque le nombre de cycles de sollicitation augmente. Cette diminution. fonction linéaire du logarithme du nombre de cycles $\mathrm{N}$. est dautant plus importante que la contrainte de

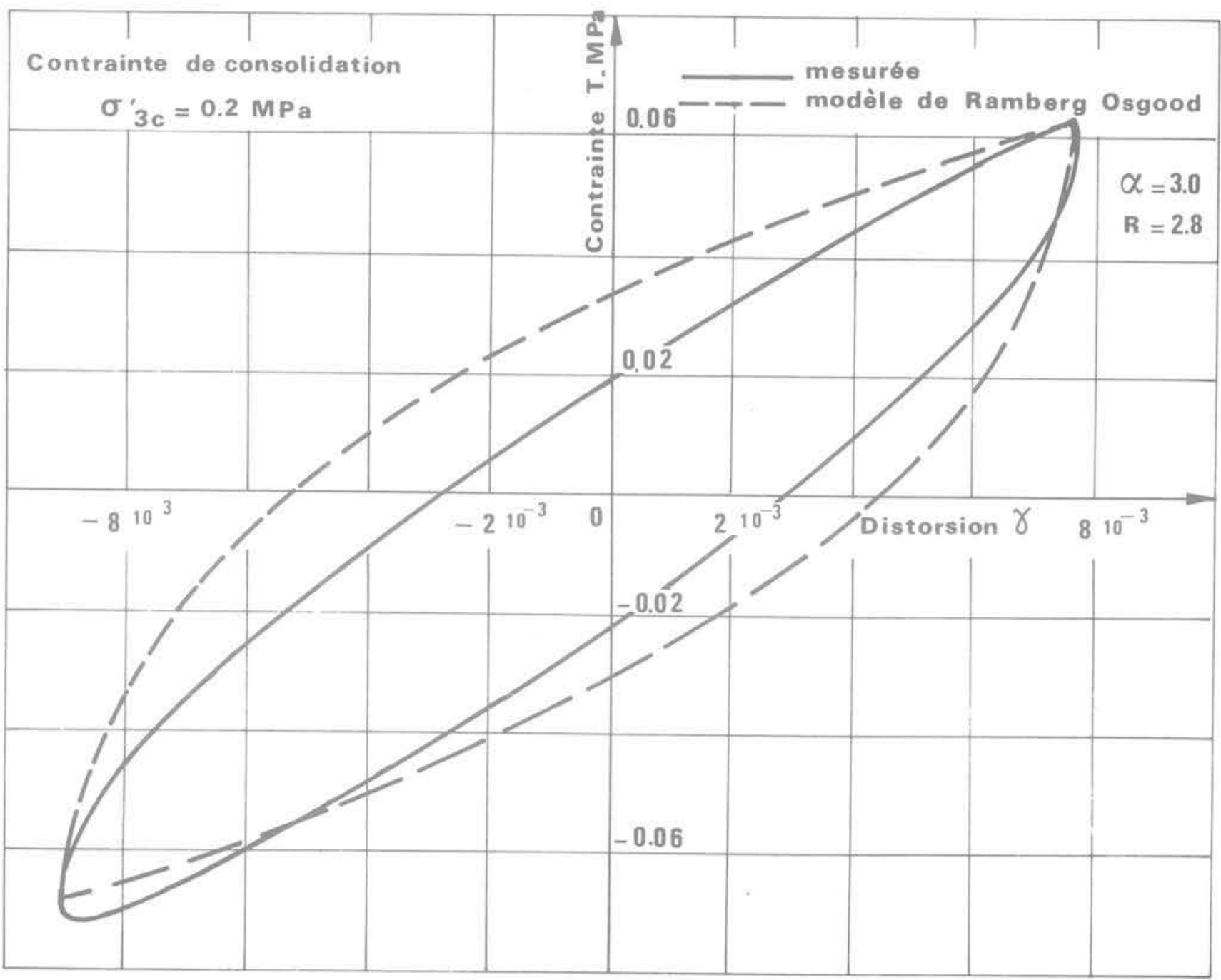

Fig. 10 Courbe d'hystérésis mesurée à l'appareil triaxial 
cisaillement cyclique est élevée. Ces résultats peuvent également être représentés dans le plan $(\tau, \gamma)$. La figure 12 donne la courbe effort déformation correspondant au premier cycle de sollicitation et celle correspondant au millième cycle. A titre de comparaison, on a également représenté la courbe théorique déduite du modèle de Ramberg-Osgood pour les valeurs des paramètres précédemment déterminés. On constate que :

- les points expérimentaux correspondant à $\mathrm{N} 1$ cycle sont très voisins de la courbe théorique pour les plus faibles valeurs de la contrainte de cisaillement; le modèle permet de définir correctement la courbe de premier chargement,

- le point expérimental correspondant à $\mathrm{N}=1$ cycle pour la valeur la plus élevée de la contrainte de cisaillement n'est plus situé sur la courbe théorique. Ceci traduit l'influence des sollicitations antérieures qui, bien qu'à des niveaux de contrainte plus faibles, ont affecté le sol,

- la courbe effort déformation pour $N=1000$ cycles est nettement différente de la courbe $N=1$ cycle, mettant en évidence une "dégradation" du sol au cours de la sollicitation. Cette dégradation n'est pas totalement irréversible, car, au niveau de contrainte supérieur, le point correspondant à $N=1$ cycle se rapproche à nouveau de la courbe théorique de premier chargement, l'écart étant cependant d'autant plus important que le niveau de contrainte est plus élevé.

Les résultats de tous les essais sont rassemblés sur la figure 13 qui donne, pour une déformation $\gamma$, le rapport $\delta$ entre la contrainte de cisaillement correspondant à la courbe $\mathrm{N}=1000$ cycles et celle correspondant à la courbe $\mathrm{N}=1$ cycle. Ce paramètre de dégradation introduit par Idriss (1979) est aussi égal

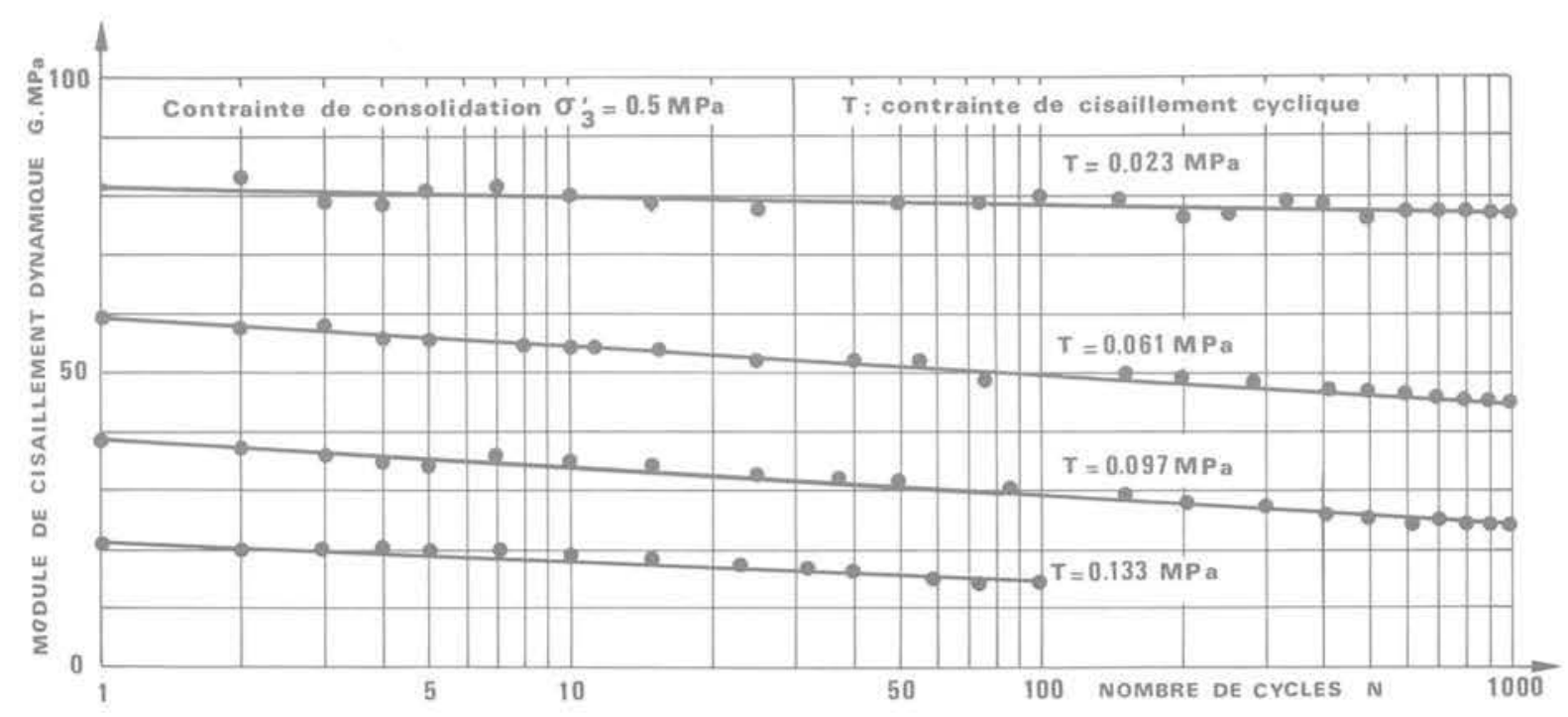

Fig. 11 Essai de fatigue à l'appareil triaxial

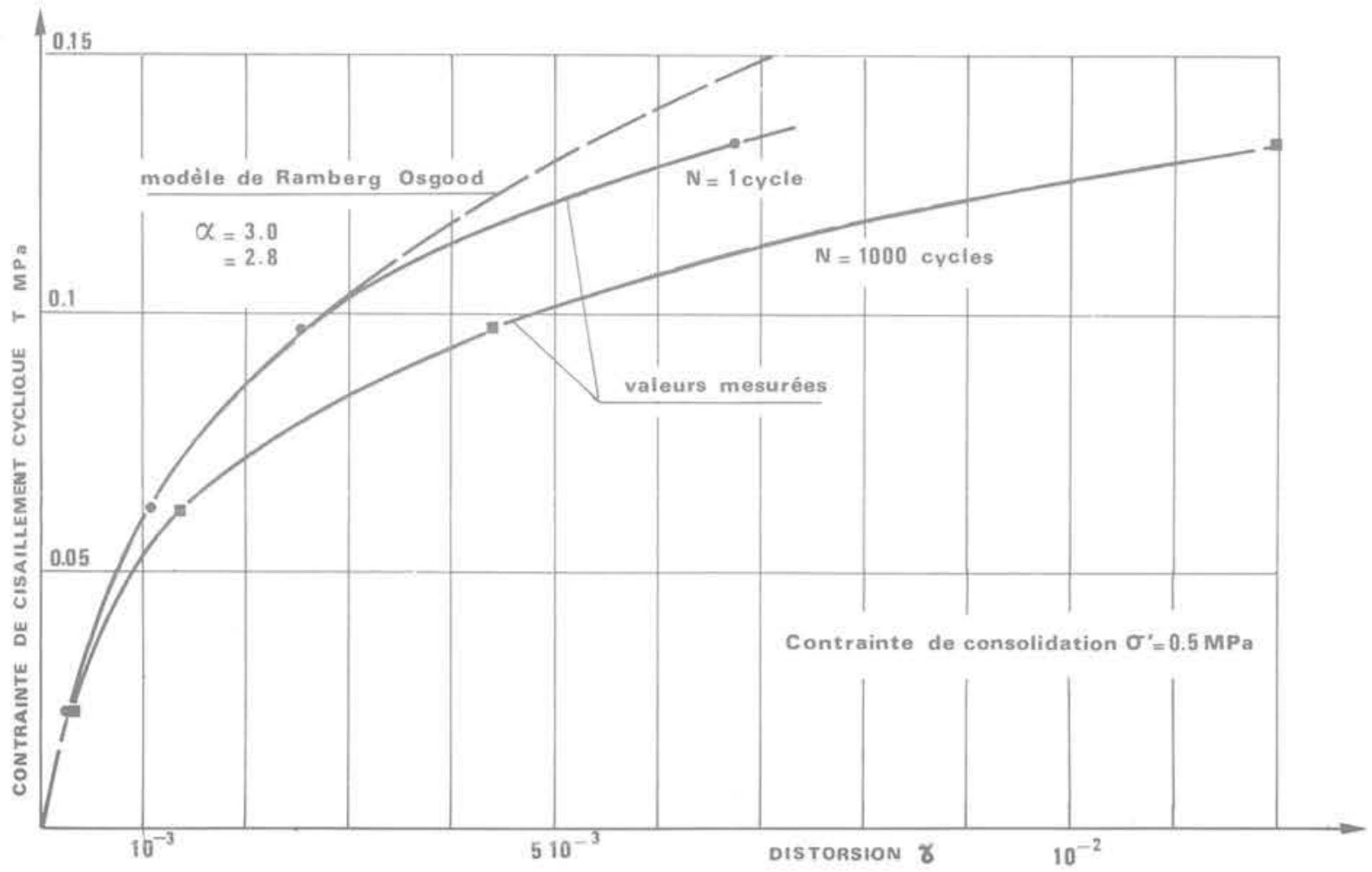

Fig. 12 Essai de fatigue à l'appareil triaxial 
au rapport des modules à 1000 cycles et à 1 cycle. A forte déformation, I'utilisation des courbes expérimentales surestime la valeur de $\varepsilon$. Si ce rapport est calculé à partir de la courbe expérimentale $\mathrm{N}=1000 \mathrm{cy}$ cles et de la courbe théorique $\mathrm{N}=1$ cycle, la dispersion est plus faible. La droite $(\Delta)$ de la figure 13 représente correctement la diminution moyenne de module entre le premier et le millième cycle. Pour une déformation de $510^{-3}$, le module au millième cycle représente $70 \%$ du module au premier cycle à cette même déformation.

Lors d'études antérieures, il a été constaté qu'une interruption de quelques heures de la sollicitation cyclique se traduisait à drainage fermé par un accroissement du module. Pour étudier plus systématiquement ce phénomène, on a réalisé des essais de fatigue entrecoupés de périodes de repos. Sur chaque éprouvette, on a réalisé la séquence d'opérations suivantes : application de $\mathrm{N}$ cycles de sollicitation à un niveau de contrainte $\tau$ donné - arrêt de la sollicitation au temps $t_{0}-$ mesure des modules au temps $t_{0}+15$ minutes, $t_{0}+30$ minutes, $t_{0}+1$ heure, $t_{0}+2$ heures, $t_{0}+4$ heures, $t_{0}+6$ heures, $t_{0}+8$ heures et $t_{0}+24$ heures. Pour chaque éprouvette, on a réalisé à un niveau de contrainte $\tau$ donné, trois séquences successives de 100, 200 et 700 cycles. Après la dernière séquence, une valeur du module a été mesurée après 48 heures de repos. A aucun moment le drainage n'est ouvert. Après la dernière séquence de sollicitation, les éprouvettes sont écrasées à drainage fermé.

Un résultat type est donné sur la figure 14. On constate que :

- aux faibles valeurs de la contrainte de cisaillement cyclique, la décroissance du module avec le nombre de cycles suit la même loi, à la dispersion près, lors de la première série de 100 cycles ou lors des séries consécutives de 200 et 700 cycles imposées après des périodes d'interruption de 24 heures.

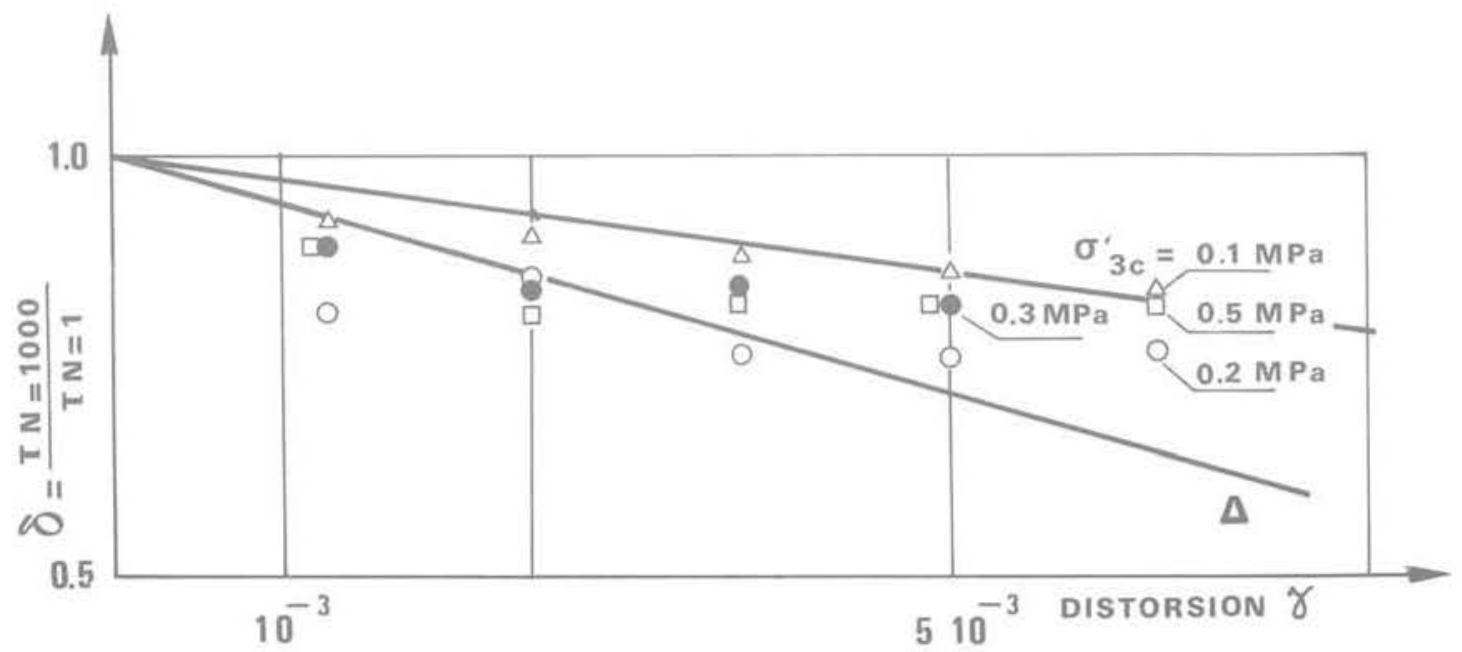

Fig. 13 Essais de fatigue à l'appareil triaxial - Paramètre de dégradation

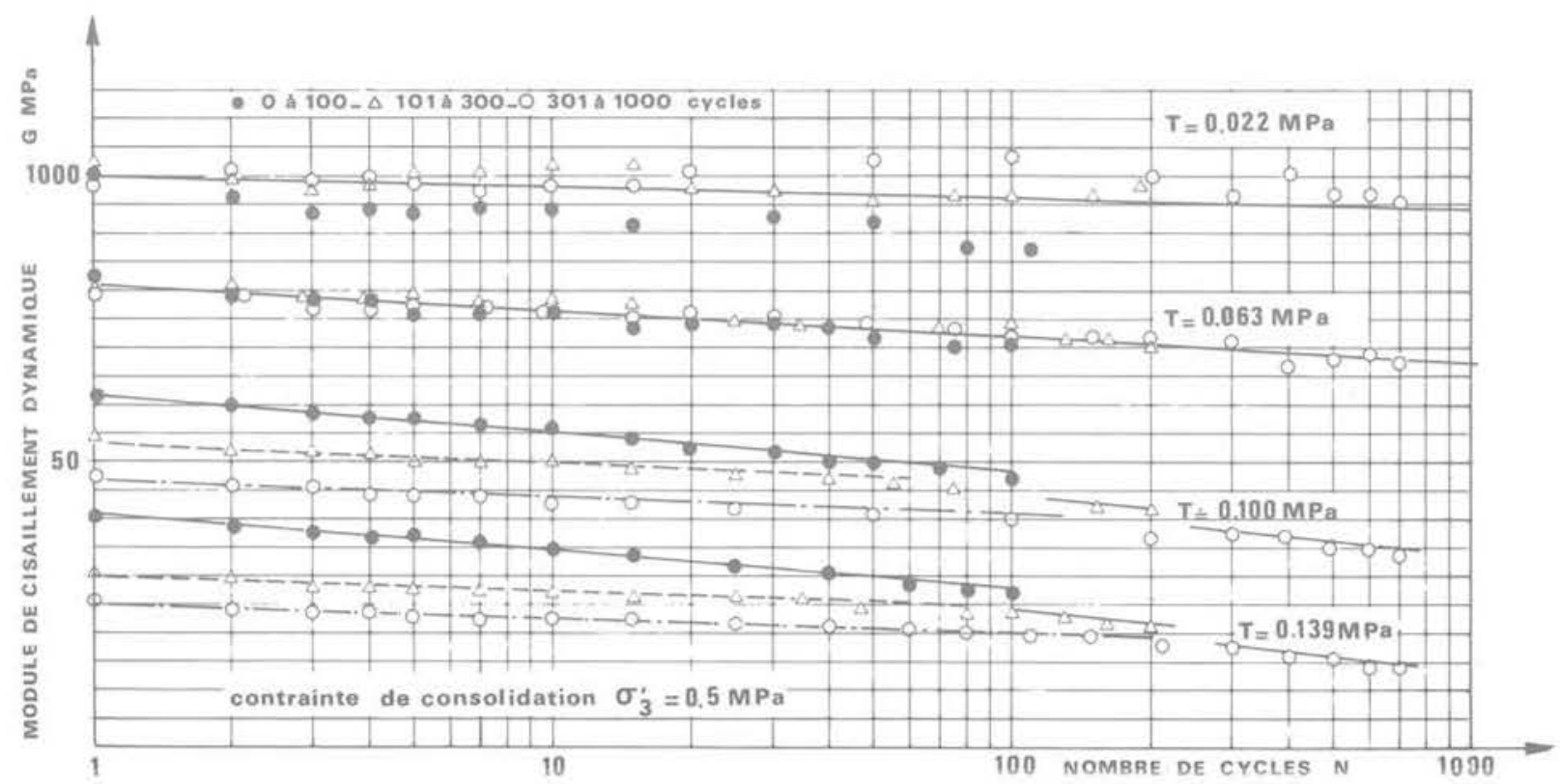

Fig. 14 Essai de fatigue à l'appareil triaxial avec période de repos entre les séries de cycles 


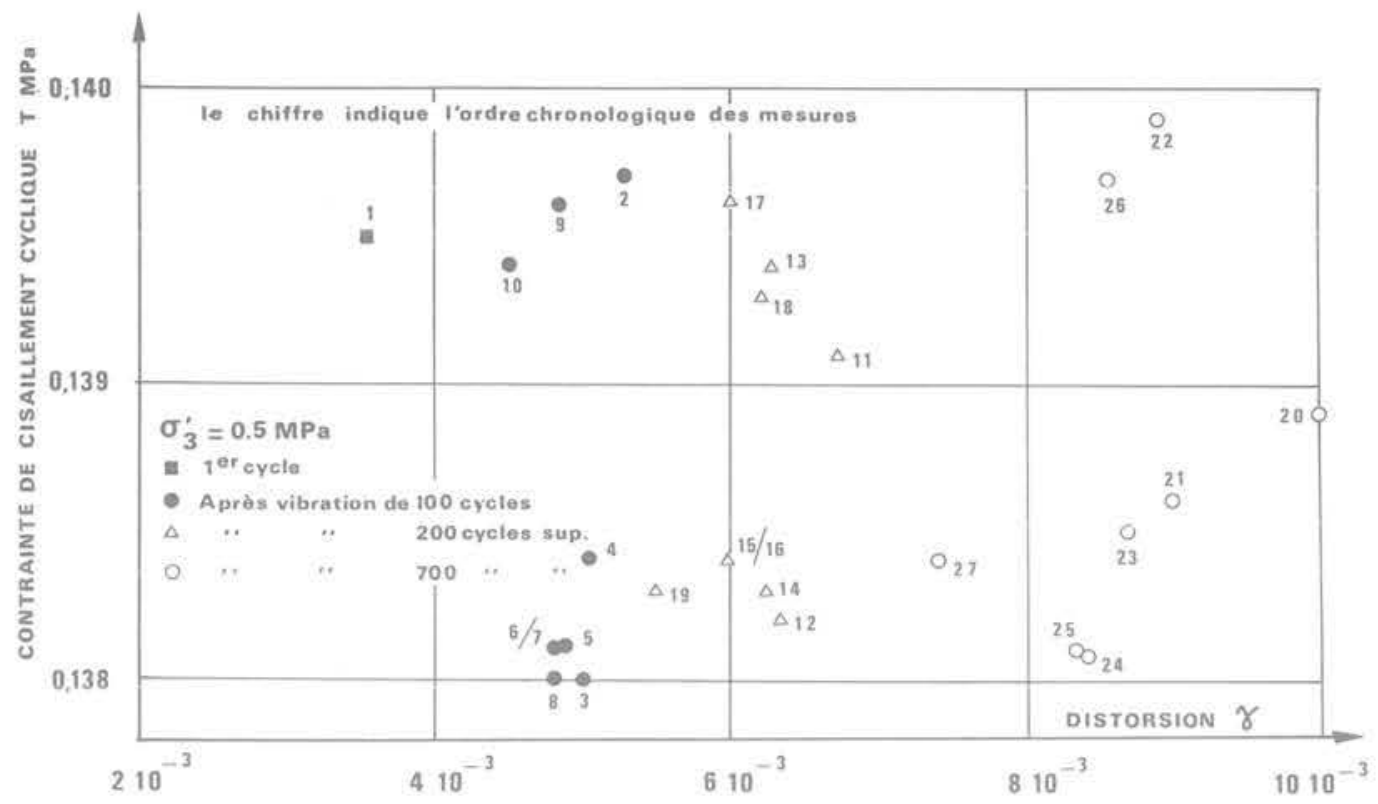

Fig. 15 Essai de fatigue à l'appareil triaxial - Regain du module avec le temps $G=T / \gamma$

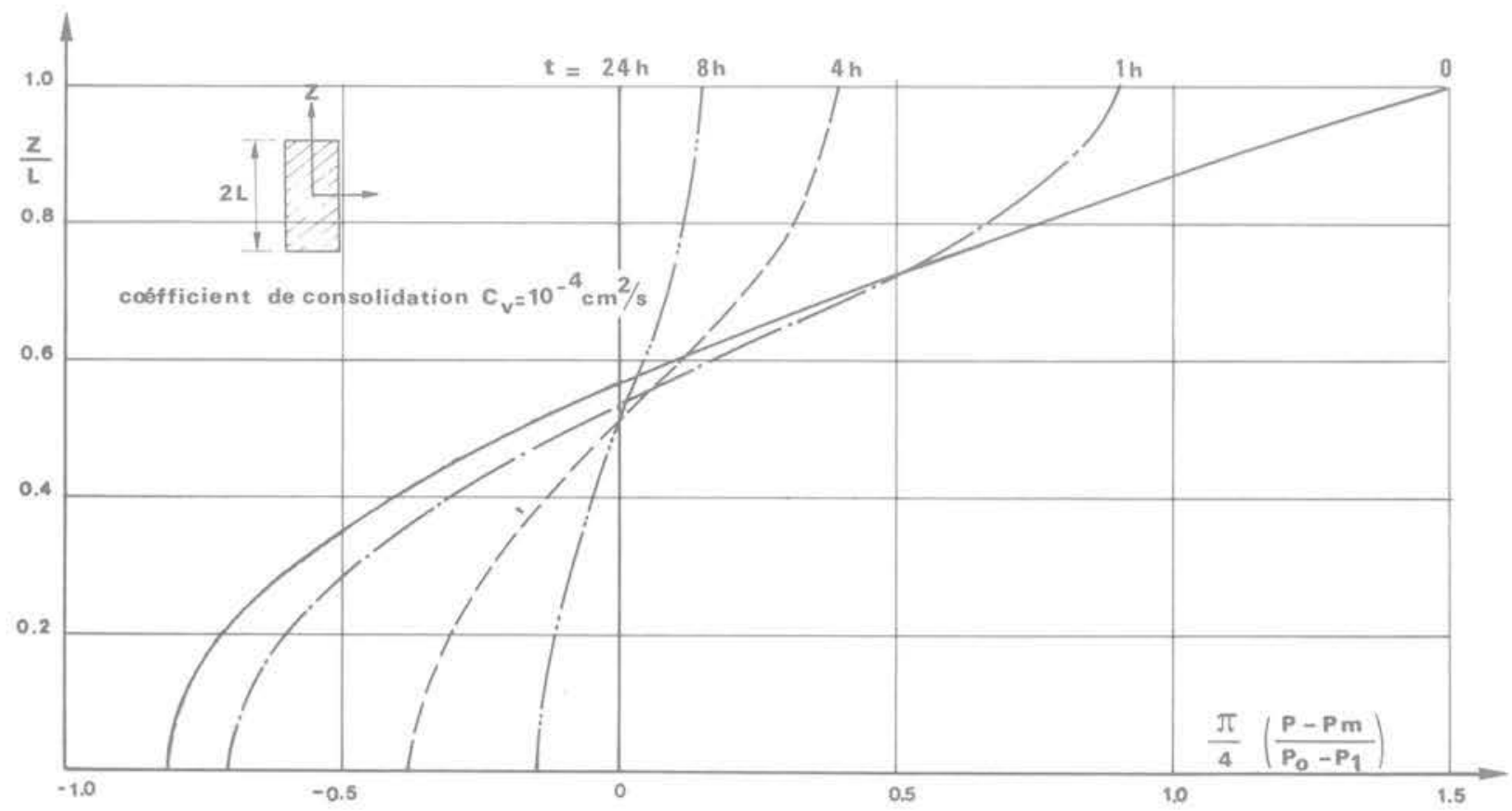

Fig. 16 Evolution de la répartition des pressions interstitielles dans une éprouvette (drainage fermé)

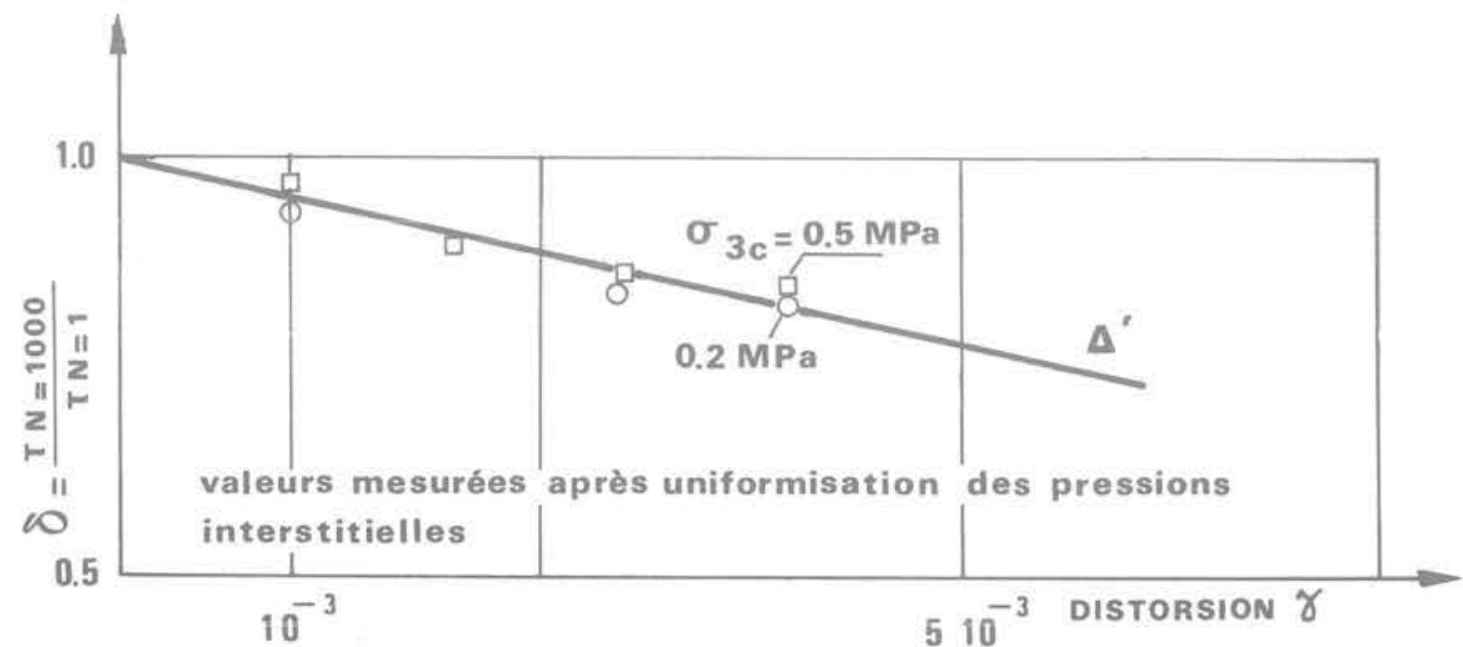

Fig. 17 Essai de fatigue à l'appareil triaxial - Paramètre de dégradation 
- aux valeurs plus élevées de la contrainte de cisaillement, la décroissance du module avec le nombre de cycles est plus faible lors des sollicitations ultérieures tant que le nombre de cycles reste inférieur au nombre de cycles $N_{0}$ de la sollicitation antérieure (courbe en tiretés de la figure 14). Au-delà de $N_{0}$, le taux de décroissance redevient sensiblement le même que celui mesuré lors de la première série de cycles. Le module, après une période de repos de 24 heures, est supérieur à celui mesuré en fin de sollicitation tout en restant inférieur à sa valeur initiale.

Pour mieux apprécier l'augmentation du module dynamique dans le temps, après l'interruption de la sollicitation cyclique, on a représenté (fig. 15), dans le plan $(\tau, \gamma)$, les points correspondant aux mesures suivantes : premier cycle de la sollicitation - dernier cycle de la sollicitation, - mesure après 15 minutes, 30 minutes, 1 heure, 2 heures, 4 heures, 8 heures et 24 heures de repos. Le point 1 est sur la courbe de premier chargement. Le point 2 correspond à la valeur mesurée au centième cycle et se déduit du point 1 par une translation dans le sens des $\gamma$ croissants. Au cours du temps, le point représentatif se déplace dans le plan $(\tau, \gamma)$ [points 3,4 , etc.] en se rapprochant généralement de la courbe de premier chargement, ce qui traduit un accroissement du module. Une nouvelle sollicitation déplace à nouveau le point dans le sens des $\gamma$ croissants (point 11 mesuré après 200 cycies supplémentaires). Le même phénomène d'accroissement du module est de nouveau observé (points 12 à 19). Le temps nécessaire à la stabilisation du module est variable suivant l'étreinte de consolidation et l'amplitude de la sollicitation.

D'une façon générale, les constatations précédentes sont valables pour l'ensemble des essais même si, pour certains d'entre eux, la dispersion des mesures est importante.

Le drainage étant fermé pendant les phases de repos, l'accroissement du module de cisaillement dynamique ne peut pas résulter d'une reconsolidation avec variation de l'indice des vides. Pour l'expliquer, l'hypothèse faite est la suivante : la sollicitation dynamique appliquée par l'intermédiaire d'un piston, sollicitant en compression et décompression l'échantillon, créé au voisinage des têtes des concentrations de contrainte importantes entraînant une augmentation plus forte qu'au centre de l'échantillon de la pression interstitielle. Celle-ci n'est donc pas uniforme en fin de sollicitation. Le module, fonction de la contrainte moyenne effective $\sigma_{m}^{\prime}$, est plus faible au voisinage des têtes, et ces zones à module plus faible influent sur le comportement global. En pèriode de repos, il se produit, à drainage fermé, une uniformisation de la pression interstitielle entrainant une augmentation du module vers les têtes et une diminution vers le centre. Expérimentalement, le résultat net est une augmentation du module global.

Une sollicitation cyclique de faible amplitude n'engendre que de très faibles augmentations de pression interstitielle; celle-ci, une fois uniformisée, ne modifie pratiquement pas le module (regain total de la valeur après 24 heures). Une sollicitation de forte amplitude engendre une pression interstitielle significative qui, même après uniformisation, entraîne une diminution du module (regain partiel). A ce phénomène, on pourrait également superposer une détérioration irréversible des liaisons interparticules due à la sollicita- tion cyclique. Cette détérioration n'existe vraisemblablement pas pour les essais effectués et le sol étudié, car son influence devrait apparaître sur les cohésions non drainées mesurées après sollicitation cyclique. Ce point sera examiné au paragraphe suivant.

Si l'on définit une répartition initiale de la pression interstitielle en fin de sollicitation cyclique, il est possible de calculer son évolution dans le temps à drainage fermé. On a admis une répartition initiale parabolique de valeur maximale $p_{0}$ en tête, de valeur minimale $p_{1}$ au centre et de valeur moyenne $p_{m}$. La variation de $\left(p-p_{m}\right) /\left(p_{0}-p_{1}\right)$ est donnée figure 16 pour un coefficient de consolidation de $C_{v}=10^{-4} \mathrm{~cm}^{2} / \mathrm{s}$ correspondant à la valeur mesurée en décharge lors de l'essai œdométrique. En effet, lors de la sollicitation dynamique, la contrainte intergranulaire diminue par augmentation de la pression interstitielle, et ce phénomène est plus assimilable à un déchargement qu'à un chargement. D'après les résultats prèsentès sur la figure 16, une période de 24 heures serait nécessaire pour permettre une uniformisation de la pression interstitielle. Ce résultat est en bon accord avec les constatations expérimentales. II resterait à vérifier l'hypothèse de répartition initiale de la pression au moyen de capteurs placés au centre et à la base de l'échantillon.

Avec l'hypothèse faite, le comportement réel du sol est représenté par les modules mesurés après uniformisation de la pression interstitielle. Pour tous les essais réalisés, la figure 17 donne la variation du paramètre de dégradation \& entre le premier et le millième cycle. On constate que :

- la diminution du module ne dépend pas de l'étreinte de consolidation mais seulement de la déformation.

- la dispersion est nettement plus faible que précédemment,

- la diminution du module est évidemment moins importante. La pente de la droite $\left(\Delta^{\prime}\right)$ dépend du nombre de cycles (N). L'interprétation de tous les essais réalisés conduit à écrire :

$$
\delta=1-b y \log N
$$

où b est une constante expérimentale; dans le cas présent $\mathrm{b}=15$.

On peut modifier la formulation de la loi de comportement pour tenir compte de la dégradation du module avec l'augmentation du nombre de cycles de sollicitation. Le comportement de la vase du Blayais sous chargement cyclique est alors défini par les 3 équations :

$$
\begin{gathered}
\gamma=\gamma_{c}+\left[\frac{\tau-\tau_{c}}{\delta}\right] \frac{1}{G_{\text {max }}}\left[1+H\left(\frac{\left|\tau-\tau_{c}\right|}{n}\right)\right] \\
H(x)=\alpha\left[\frac{x}{\delta \tau_{y}}\right]^{R-1} \\
\delta=1-b \gamma \log N
\end{gathered}
$$

avec

$$
\begin{aligned}
& G_{\text {max }}=210 \mathrm{p}_{\mathrm{a}}\left[\frac{\sigma_{\mathrm{m}}^{\prime}}{\mathrm{p}_{\mathrm{a}}}\right]^{0,9} \\
\alpha & =3,0 \\
\mathrm{R} & =2,8 \\
\mathrm{~b} & =15 \\
\tau_{\mathrm{y}} & =\text { cisaillement maximal. }
\end{aligned}
$$




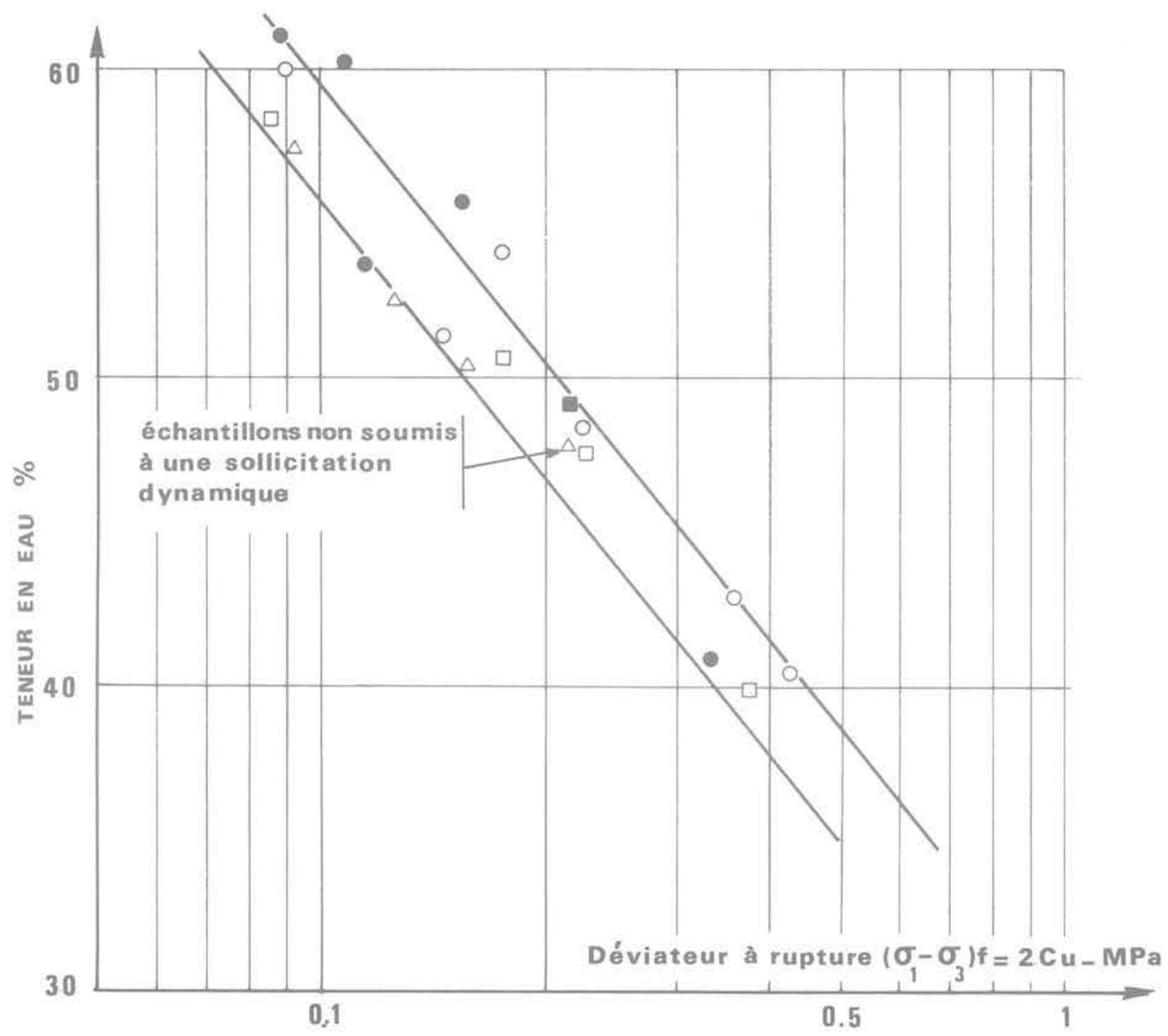

Fig. 18 Variation de la résistance au cisaillement non drainée avec la teneur en eau après sollicitation dynamique

\section{Caractéristiques mécaniques apparentes après sollicitation cyclique}

Après chaque essai dynamique (triaxial, colonne résonnante ou essai de fatigue), les échantillons ont été cisaillés à drainage fermé. Les cohésions apparentes mesurées sont reportées sur la figure 18 en fonction de la teneur en eau. On a également reporté, pour permettre la comparaison, les résultats d'un essai triaxial consolidé non drainé (CU). A la dispersion près (pouvant provenir de légères différences de teneur en matière organique), on peut admettre qu'il $y$ a une relation bi-univoque entre la teneur en eau et la cohésion non drainée, et conclure que les sollicitations cycliques n'ont pas affecté les caractéristiques mécaniques du sol. Cette conclusion n'est valable que pour le programme réalisé où le nombre de cycles de sollicitation à forte déformation (de l'ordre de $10^{-2}$ ) est relativement faible. D'autres études ont en effet montré (Seed 1968) que la résistance au cisaillement apparente pouvaít diminuer après des sollicitations répétées de forte amplitude. Comme les caractéristiques mécaniques apparentes n'ont pas varié, les diminutions de module observées sous sollicitation de longue durée ne peuvent pas provenir d'une détérioration irréversible des liaisons interparticules du sol qui entraînerait une variation de sa cohésion apparente. L'hypothèse faite, consistant à attribuer la diminution de module à une augmentation de pression interstitielle, semble donc justifiée.

\section{Conclusions}

La présente étude du comportement, sous chargement cyclique, des vases normalement consolidées du site du Blayais, permet de tirer les conclusions suivantes :

- le comportement sous sollicitation cyclique unidirectionnelle et d'amplitude constante peut être modélisé à l'aide du modèle de Ramberg-Osgood dérivé des lois de Masing. La courbe de premier chargement est correctement modélisée mais l'hystérésis du sol à forte déformation est surévalué. On peut envisager de modifier la deuxième loi de Masing pour diminuer I'hystérésis attribué par le modèle au matériau;

- les diminutions de module, mesurées lors d'essai de fatigue à l'appareil triaxial dynamique et en l'absence de période de repos, sont surestimées par suite d'une répartition non uniforme des pressions interstitielles. Elles ne traduisent pas le comportement réel du sol:

- les diminutions effectives de modules, mesurées lors d'essai de fatigue à l'appareil triaxial dynamique 
après uniformisation des pressions interstitielles, peuvent être principalement attribuées, pour des déformations faibles à moyennes, à une augmentation de pression interstitielle créée par la sollicitation cyclique. Ce comportement peut être pris en compte en modifiant la formulation du modèle de Ramberg-Osgood par l'introduction d'un paramètre de dégradation ne dépendant que de la déformation maximale et du nombre de cycles;

- la résistance au cisaillement apparente du sol n'est pas affectée par des sollicitations cycliques, même en grand nombre, d'amplitude faible à moyenne.

\section{Remerciements}

Cette étude a été financée par Mécasol avec le concours de la D.G.R.S.T. (décision d'aide $n^{0} 78.7 .0 .240$ ) dont le support est vivement remercié. Les échantillons ayant servi à l'étude ont été fournis par la Région d'Équipement Paris de I'E. D.F. que nous remercions.

\section{Références}

HARDIN B. O. (1968) «Vibrations of Normally Consolidated Clays ".

BLACK W. L. Journal of Soil Mechanics and Foundations Division A. S. C.E. - Vol. 94. n० SM2.

HARDIN B. O. (1978) "The nature of Stress Strain Behavior for Soils". Proc. A.S. C.E. Geotechnical Engineering Division Specialty Conference on Earthquake Engineering and Soil Dynamics - Pasadena California.

MASING G. (1926) "Eigenspannungen und Verfestigung beim Messing ". Proc. 2nd International Congress of Applied Mechanics.
PECKER A. (1979) *Comportement Dynamique des Sols ". Séminaire Séismes - Sols - Structures I.T.B.T.P. Collège International des Sciences de la Construction - Nov. 1979.

RICHART F. E., HALL J. R., WOODS R. D. (1970), "Vibrations of Soils and Foundations". Prentice Hall Inc.

THIERS G. R., SEED H. B. (1968) "Strength and Stress Strain Characteristics of Clays subjected to Seismic Loading Conditions ". Vibration effects of earthquakes on soils and foundations A.S.T.M. - STP 450 American Society for testing and Materials.

\section{Notations}

b constante expérimentale (éq. 11)

$\mathrm{C}_{u} \quad$ cohésion non drainée

G module de cisaillement tangent

Gs module de cisaillement sécant

$\mathrm{G}_{\max }$ module de cisaillement maximal

n paramètre de la loi de Ramberg-Osgood (éq. 6)

$\mathrm{N}$ nombre de cycles

pa pression atmosphérique

R paramètre de la loi de Ramberg-Osgood (éq. 7)

a paramètre de la loi de Ramberg-Osgood (éq. 7)

$\beta$ pourcentage d'amortissement critique équivalent (éq. 3)

y déformation de cisaillement

$\dot{\gamma}$ vitesse de déformation de cisaillement

$\delta$ paramètre de dégradation (éq. 13)

$\eta \quad$ coefficient de viscosité

$r_{m}^{\prime} \quad$ contrainte moyenne effective de consolidation

$\tau \quad$ contrainte de cisaillement cyclique

$\tau_{y} \quad$ contrainte de cisaillement de référence.

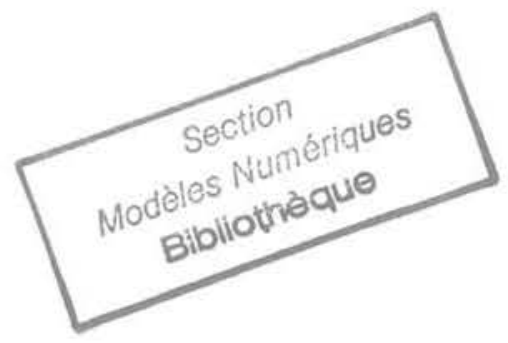


